\title{
Hemodynamic analysis of the recipient parasylvian cortical arteries for predicting postoperative hyperperfusion during STA-MCA bypass in adult patients with moyamoya disease
}

\author{
Jianjian Zhang, MD, PhD, ${ }^{1}$ Sirui Li, MD, PhD, ${ }^{2}$ Miki Fujimura, MD, PhD, ${ }^{3}$ Tsz Yeung Lau, MD, PhD, ${ }^{4}$ \\ Xiaolin Wu, MD, PhD, ${ }^{1}$ Miao Hu, PhD, ${ }^{1}$ Hanpei Zheng, MD, PhD, ${ }^{2}$ Haibo Xu, MD, PhD, ${ }^{2}$ \\ Wenyuan Zhao, MD, PhD, ${ }^{1}$ Xiang $\mathrm{Li}, \mathrm{PhD},{ }^{5}$ and Jincao Chen, MD, PhD ${ }^{1}$
}

1Department of Neurosurgery, Zhongnan Hospital of Wuhan University; ${ }^{2}$ Department of Radiology, Zhongnan Hospital of Wuhan University, Wuhan, China; ${ }^{3}$ Department of Neurosurgery, Kohnan Hospital, Sendai, Japan; ${ }^{4}$ Department of Neurosurgery, Lakeland Regional Hospital, University of South Florida, Lakeland, Florida; and ${ }^{5}$ Queensland Brain Institute, University of Queensland, Brisbane, Queensland, Australia

OBJECTIVE Superficial temporal artery-middle cerebral artery (STA-MCA) bypass is a common approach for treating moyamoya disease (MMD); however, the selection of recipient vessels is still controversial, and its relationship with postoperative cerebral hyperperfusion (CHP) has not been revealed. The aim of the study was to investigate the relationship between the hemodynamic sources of the recipient parasylvian cortical arteries (PSCAs) and the occurrence of postoperative CHP.

METHODS The authors retrospectively analyzed the clinical data from 68 adult patients (75 hemispheres) with MMD who underwent STA-MCA bypass. Based on their hemodynamic sources from the MCA and non-MCAs, the PSCAs were classified as M-PSCAs and non-M-PSCAs, and their distributional characteristics were studied. Moreover, the patients' demographics, incidence of postoperative CHP, and post- and preoperative relative cerebral blood flow values were examined.

RESULTS The digital subtraction angiography analysis demonstrated that $40 \%(30 / 75)$ of the recipient PSCAs had no hemodynamic relationship with the MCA. The post- and preoperative relative cerebral blood flow values of the M-PSCA group were significantly higher than those of the non-M-PSCA group $(p<0.001)$. Multivariate analysis revealed that the hemodynamic source of PSCAs from the MCA was significantly associated with the development of focal $(p=0.003)$ and symptomatic $(p=0.021)$ CHP. Twelve $(85.7 \%)$ of the 14 patients with symptomatic CHP and all $4(100 \%)$ patients with postoperative hemorrhage were from the M-PSCA group.

CONCLUSIONS This study revealed that direct anastomoses of PSCAs with anterograde hemodynamic sources from the MCA had a high risk of postoperative CHP during STA-MCA bypass in adult patients with MMD.

https://thejns.org/doi/abs/10.3171/2019.10.JNS191207

KEYWORDS moyamoya disease; cerebral hyperperfusion; cerebral blood flow; hemodynamics; bypass surgery; vascular disorders

$\mathrm{M}$ OYAMOYA disease (MMD) is a chronic, occlusive cerebrovascular disease of unknown etiology characterized by bilateral stenotic-occlusive changes at the terminal portion of the internal carotid artery (ICA) and an abnormal vascular network at the base of the brain. ${ }^{5,24,26}$ Extracranial-intracranial bypass, such as superficial temporal artery-middle cerebral artery (STAMCA) anastomosis, has a generally favorable long-term outcome for patients with ischemic MMD, and has a positive effect on hemorrhagic-onset MMD according to the

ABBREVIATIONS $\mathrm{ACA}=$ anterior cerebral artery; $\mathrm{CBF}=$ cerebral blood flow; $\mathrm{CHP}=$ cerebral hyperperfusion; $\mathrm{CTA}=\mathrm{CT}$ angiography; $\mathrm{CTP}=\mathrm{CT}$ perfusion; $\mathrm{DSA}=$ digital subtraction angiography; ECA = external carotid artery; ICA = internal carotid artery; $M C A=$ middle cerebral artery; $M M D=$ moyamoya disease; $M T T=$ mean transit time; $\mathrm{PCA}=$ posterior cerebral artery; PSCA = parasylvian cortical artery; $\mathrm{rCBF}=$ relative $\mathrm{CBF} ; \mathrm{STA}-\mathrm{MCA}=$ superficial temporal artery-MCA; TND = transient neurological deficit. SUBMITTED May 2, 2019. ACCEPTED October 8, 2019.

INCLUDE WHEN CITING Published online December 27, 2019; DOI: 10.3171/2019.10.JNS191207. 

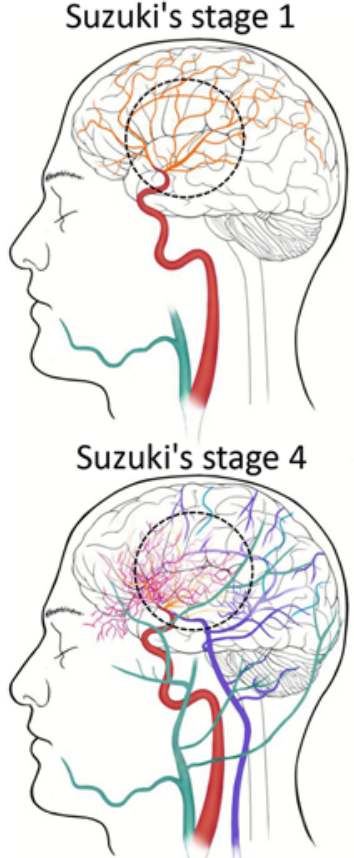

ICA $\square$ MCA $\square$ Moyamoya
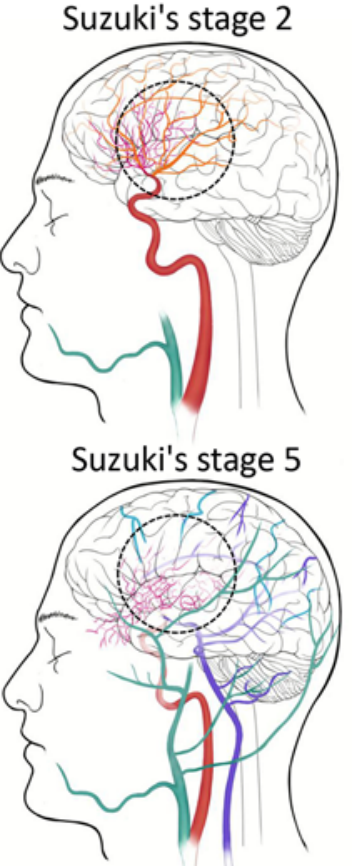
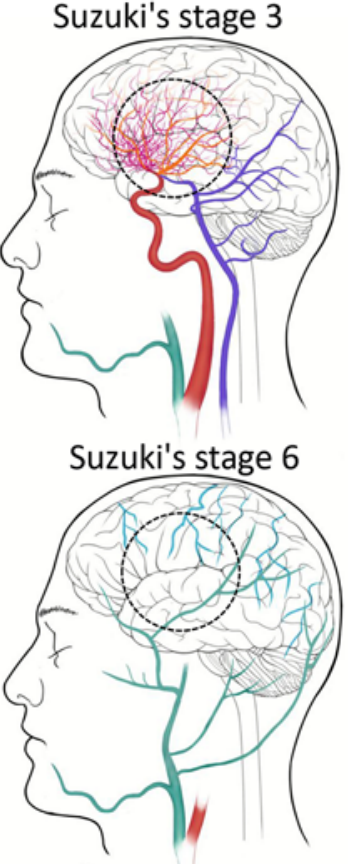

FIG. 1. Different hemodynamic sources of the PSCAs with different Suzuki angiographic stages. As the scores of Suzuki angiographic staging increased, the number of PSCAs with anterograde hemodynamic sources from the MCA decreased. The blood supplies of the suitable recipient PSCAs (black circle) for anastomosis may come completely from non-MCAs. collateral.br = collateral branches. Copyright Jiayi Chen and Jianjian Zhang. Published with permission.

Japan Adult Moyamoya (JAM) Trial. ${ }^{710,20,23}$ However, one of the common issues with direct bypass surgery is cerebral hyperperfusion (CHP), which can cause severe complications, such as transient neurological deficits (TNDs) and intracranial hemorrhage. . $, 3,14,16,27,28$ The occurrence of postoperative CHP is still not fully understood and is difficult to accurately predict through diagnosis prior to surgery.

Direct cerebral bypass surgery can be viewed as a "battle" between the extracranial and intracranial circulation due to blood flow competition between the donor and recipient arteries. ${ }^{9,12}$ Preoperative analysis of the hemodynamic characteristics of recipient vessels can provide an important understanding of the effects of the revascularization procedure. In STA-MCA bypass surgeries, the recipient vessels are typically the distal branches of the MCA, such as $\mathbf{M}_{4}{ }^{5,7,10,20,23,24}$ However, as Suzuki's angiographic staging increases, the number of parasylvian cortical arteries (PSCAs) with anterograde hemodynamic sources from the MCA decreases (Fig. 1). Because collateral blood flow is common in patients with MMD, ${ }^{4,25}$ the cortical branches of the MCA may shrink, and suitable recipient PSCAs for anastomosis can be the dilated distal branches of non-MCAs. Furthermore, even though all of the PSCAs are anatomical branches of the MCA, their major source of blood flow is not necessarily from the MCA.

Because the differences between patients with MCA and non-MCA blood supplies in terms of the incidence of CHP may possibly be related to the degree of hemodynamic failure, we hypothesized that in patients with MCA supply, proximal severe stenosis with moyamoya-like changes would lead to a higher degree of hypoperfusion compared to patients with non-MCA supply with good collaterals and better perfusion. Previous studies have suggested that with an increasing degree of MCA stenosis arteriolar function becomes more impaired, ${ }^{8}$ and distal to hemodynamically relevant stenosis the velocity usually dampens as well. ${ }^{17}$ Therefore, it has been speculated that the blood flow velocity, blood pressure, autoregulation ability, and even arterial quality of recipient cortical arteries with their blood supply from the MCA are much lower than in those with blood supply from non-MCAs in MMD.

In this study we analyzed the hemodynamic sources of the PSCAs, which are commonly selected as recipient vessels, in 68 adult patients (75 hemispheres) with MMD. The objective of the study was to investigate the relationship between the hemodynamic sources of recipient PSCAs and postoperative CHP.

\section{Methods \\ Inclusion Criteria}

We retrospectively collected and analyzed the data from a case series of adult patients with MMD who underwent standard STA-MCA bypass combined with encephaloduromyosynangiosis (EDMS) revascularization by 2 surgeons (J.J.Z. or J.C.C.) in our hospital between 
January 2017 and November 2018. All patients satisfied the diagnostic criteria of the Research Committee on Spontaneous Occlusion of the Circle of Willis of the Ministry of Health, Labor, and Welfare, Japan. ${ }^{5,24}$ The inclusion criteria of this study were as follows: 1) the diagnosis for each patient was established by classic 6-vessel digital subtraction angiography (DSA) with 3D videos; 2) at least 2 CT perfusion (CTP) scans were performed before surgery and within 1 week after surgery in each patient to evaluate the hemodynamic condition of the surgical hemisphere; and 3) the patency of the anastomosis was confirmed by a postoperative CT angiography (CTA) scan. All hemispheres that did not match these criteria were excluded from this study. This study protocol was approved by the institutional review board at our hospital and was in accordance with the Declaration of Helsinki as revised in 1983. Written informed consent was obtained from all patients.

\section{Surgical Indication and Procedure}

The indication for surgery included patients with symptomatic MMD (ischemic or hemorrhagic) with apparent hemodynamic compromise. The craniotomy was designed to ensure simultaneous exposure of the PSCAs in the frontal, temporal, and parietal lobes. All the anastomoses were performed on PSCAs responsible for the hypoperfused brain. Before anastomosis, flow 800 indocyanine green (ICG) videoangiography was performed to observe the hemodynamic features of the cortical arteries, such as the blood flow direction, time to maximal fluorescence, and dissipation of blood. In most cases, the time needed for the anastomosis was less than 20 minutes, and the patency was subsequently confirmed by further flow 800 ICG videoangiography intraoperatively. After anastomosis, the dura mater flaps were turned over and the dural opening area was covered with the temporal muscle for indirect revascularization. Additional steps, such as temporal muscle dissection and removal of the inner skull layer, were performed to prevent mechanical compression of the cortex and graft.

\section{Postoperative Management}

All patients gradually recovered from anesthesia in the neurosurgical intensive care unit with strict blood pressure monitoring and control, including intermittent intravenous urapidil infusion, if necessary. Blood pressure control mainly depended on the basic blood pressure level of the patients in our study: if the patient had a history of hypertension $(\geq 140 / 90 \mathrm{~mm} \mathrm{Hg})$, the target systolic pressure was $120-140 \mathrm{~mm} \mathrm{Hg}$ to avoid the risk of ischemia; if not, his or her systolic pressure was strictly controlled to 120-130 $\mathrm{mm} \mathrm{Hg}$. Antiplatelet therapy and a hydration protocol were routinely administered for all the patients. If a patient developed a TND, both CTA and CTP were immediately obtained to analyze the patency of the anastomosis and to evaluate the perfusion status of the brain. If CHP was confirmed for the patient, strict blood pressure control and edaravone administration was scheduled. If there was no evidence of CHP, an MRI scan was performed to rule out acute infarction.

\section{Radiological Examination \\ DSA Images}

The DSA images were blindly investigated by 2 senior neurosurgeons (J.C.C. and J.J.Z.). Suzuki's angiographic stage was determined first. The anterior-posterior and lateral views of the ICA, external carotid artery (ECA), and vertebral artery (VA) injections and also the dynamic 3D DSA images were analyzed, and the major feeding artery to the frontal, temporal, and parietal PSCAs could be clearly identified. In the present study, 5 major hemodynamic sources were found: the MCA, anterior cerebral artery (ACA), contralateral ACA, posterior cerebral artery (PCA), and ECA - the PSCAs were correspondingly named M-PSCA, A-PSCA, CA-PSCA, P-PSCA, and E-PSCA (Fig. 2). If the analyses of the 2 neurosurgeons conflicted, a discussion was held to reach a consensus. For statistical purposes, the PSCAs with blood supply from the non-MCAs were collectively named non-M-PSCAs in this study. Combining the results of the preoperative dynamic 3D DSA images and postoperative CTA, the bypass site could be identified, and the major blood source of the recipient PSCAs from the MCA or non-MCAs was ultimately established in each surgical hemisphere.

\section{CTP and CTA Images}

Postprocessing of raw CTP source images was completed centrally on a dedicated workstation (IntelliSpace Portal, Philips). Perfusion analysis was performed for all data sets with the vendor-provided "brain perfusion" software. Perfusion parameter maps for cerebral blood flow (CBF), cerebral blood volume, mean transit time (MTT), and time to peak were generated. We defined prolonged MTT or reduced CBF as hypoperfusion compared to the values of the posterior circulation and contralateral hemisphere. ${ }^{19,22}$ Standardized elliptical mirrored regions of interest were drawn manually as a circle with a diameter of $1 \mathrm{~cm}$ in the cortical areas at the site of the anastomosis and the occipital lobes. The corresponding mirror sites of these regions of interest were automatically defined on the contralateral hemisphere with software. All postprocessing of the images and parameter measurements was performed by 2 experienced neuroradiologists. If the interpretations of the 2 observers conflicted, a discussion was held to reach a consensus. The relative cerebral blood flow (rCBF) value was defined as the ratio between the absolute CBF value of the anastomosis site and that of the control region in the occipital lobe. The blood flow change resulting from the bypass was demonstrated by the ratio of postoperative/preoperative rCBF. From the qualitative map of CBF, we defined focal hyperperfusion as including all of the following items: 1) the presence of a significant focal CBF increase (from the lowest preoperative rCBF value to the highest postoperative $\mathrm{rCBF}$ value of the supratentorial region of the bilateral hemispheres) at the site of the anastomosis (qualitative observation of an intense focal increase in CBF confined to one major vascular territory); 2) apparent visualization of STA-MCA bypass by CTA; and 3) the absence of other pathologies such as compression of the brain surface by the temporal muscle inserted for in- 


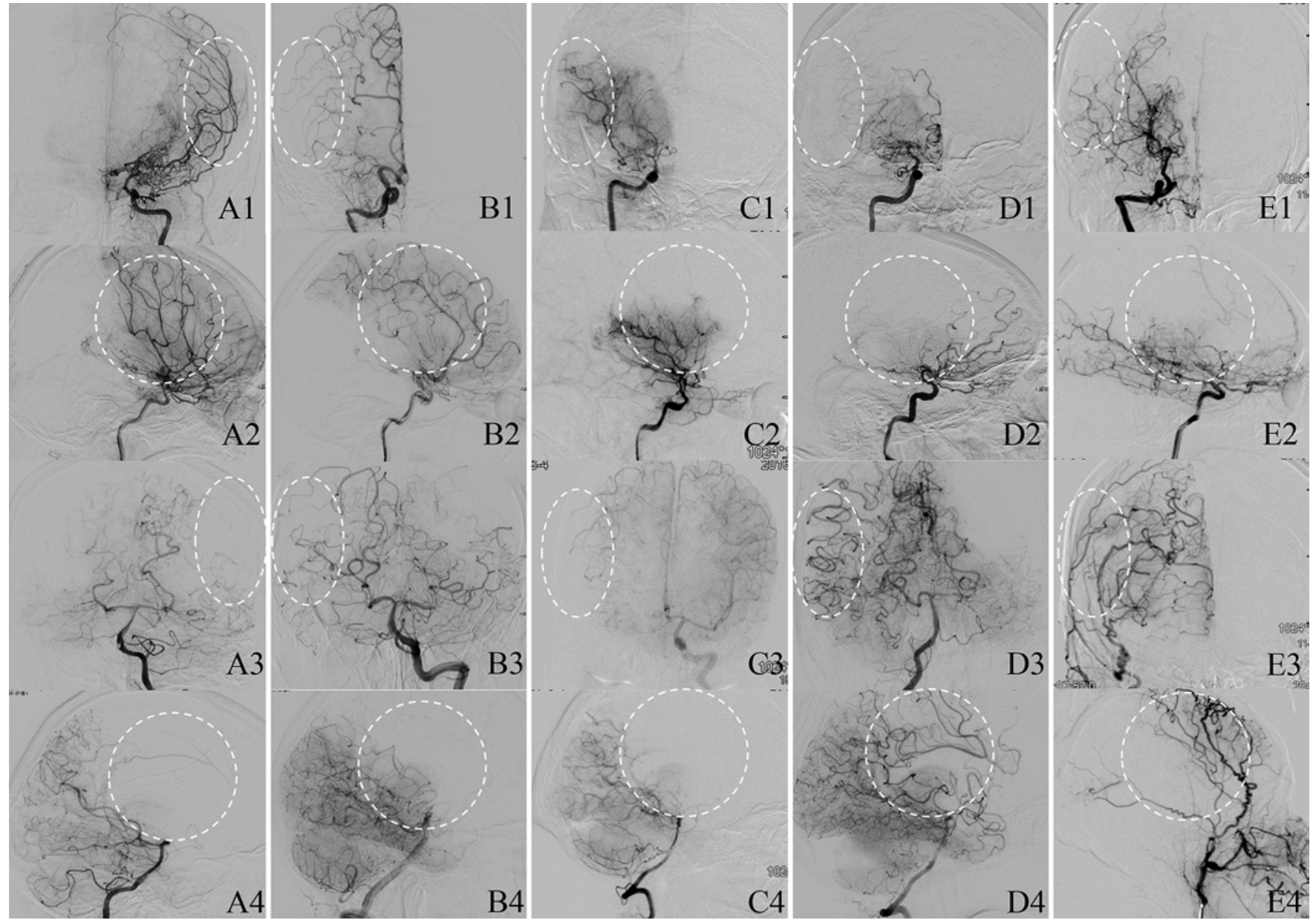

FIG. 2. Analysis of the major blood sources of the PSCAs. Representative figures of M-PSCAs and non-M-PSCAs, which are defined as the PSCAs (white circles and ovals) with blood flow from the MCA and non-MCAs. Panels A1-4 indicate the major hemodynamic sources of PSCAs originating from the MCA; B1-4 demonstrate those from the ipsilateral ACA; $1-4$ show those from the contralateral ACA; D1-4 indicate those from the PCA; and E1-4 demonstrate those from the ECA.

direct pial synangiosis, and increases in CBF secondary to seizure. ${ }^{2,3}$

\section{Statistical Analysis}

A one-way ANOVA test was used for parametric statistical analysis. Categorical variables were analyzed in contingency tables with Pearson's chi-square test and Fisher's exact test. The results with values of $p<0.05$ were considered significant. Multivariate statistical analysis of the factors related to the development of focal and symptomatic CHP, including age, sex, surgical side, onset, bypass site, and the hemodynamic source of the PSCAs, was performed using a logistic regression model. All analyses were performed with IBM SPSS Statistics Desktop, version 24 (IBM Corp.).

\section{Results}

\section{Patient Demographics}

A total of 68 consecutive adult patients (75 hemi- spheres; patient age range 18-77 years, mean 46 years) with moyamoya disease met the inclusion criteria in this study; 26 patients had hemorrhagic onset, and 42 patients had ischemic onset. Twenty-nine women (42.6\%) and 39 men $(57.4 \%)$ were enrolled.

\section{DSA Findings}

Different hemodynamic sources were observed among the frontal, temporal, and parietal PSCAs in 32 (42.7\%) hemispheres (data not shown). The distributional analysis showed that the majority of the frontal PSCAs were M-PSCAs $(38 / 75,50.7 \%)$ and A-PSCAs (17/75, 22.7\%). Similarly, most of the parietal PSCAs were also M-PSCAs (37/75, 49.3\%) and A-PSCAs (17/75, 22.7\%). In contrast to the frontal and parietal PSCAs, most of the temporal PSCAs were M-PSCAs $(50 / 75,66.7 \%)$ and P-PSCAs $(19 / 75,25.3 \%)$ (Fig. 3). In 20 hemispheres (26.7\%) the MCA did not clearly reach the cortex, as shown on DSA, and the blood flow to the PSCAs came completely from the non-MCAs (data not shown). In 


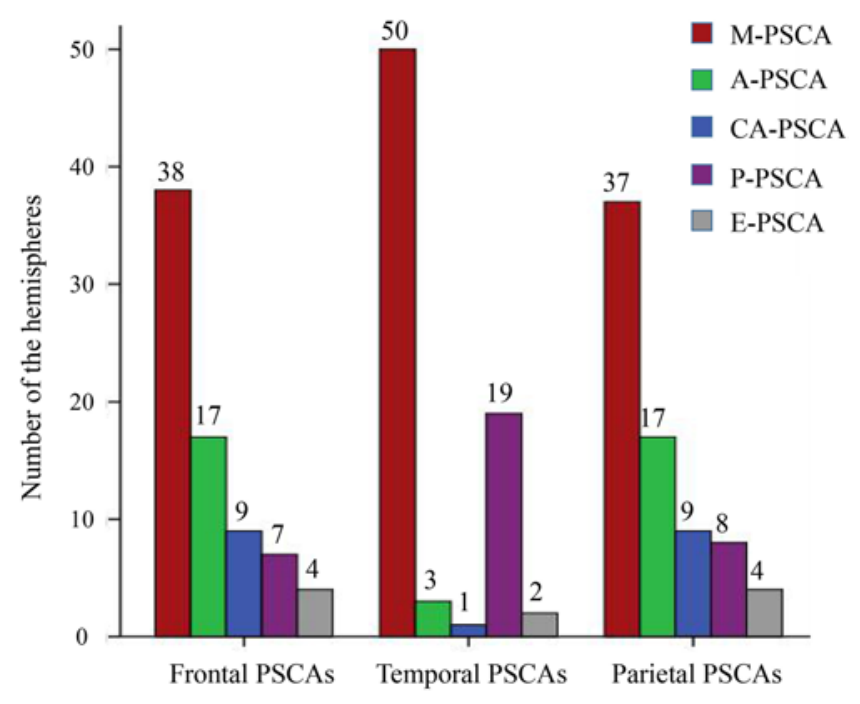

FIG. 3. Distribution of the frontal, temporal, and parietal PSCAs of the hemispheres that underwent operation according to their hemodynamic sources. Different hemodynamic sources among the frontal, temporal, and parietal PSCAs were observed in the hemispheres that underwent operation, providing the possibility of recipient artery selection. M-PSCAs, A-PSCAs, CA-PSCAs, P-PSCAs, and E-PSCAs were defined as PSCAs with blood flow from the MCA, ACA, contralateral ACA, PCA, and ECA, respectively. The distributional analysis showed that the majority of the frontal PSCAs were M-PSCAs (38/75, 50.7\%) and A-PSCAs (17/75, 22.7\%). Similarly, most of the parietal PSCAs were also M-PSCAs $(37 / 75,49.3 \%)$ and A-PSCAs (17/75, 22.7\%). In contrast to the frontal and parietal PSCAs, most of the temporal PSCAs were M-PSCAs (50/75, 66.7\%) and P-PSCAs (19/75, 25.3\%).

some cases the frontal PSCAs could even be supplied by a PCA and the temporal PSCAs by an ACA or even a contralateral ACA.

Intriguingly, only $60 \%(45 / 75)$ of the selected PSCAs were derived from the MCA, rather than $100 \%$ per the current conceptual understanding. No significant difference was observed in terms of age, sex, onset, or surgical side among the M-PSCA, A-PSCA, CA-PSCA, P-PSCA, and E-PSCA groups ( $p>0.05$ for those variables). However, the Suzuki stages of the CA-PSCA, P-PSCA, and
E-PSCA groups were significantly greater than those of the M-PSCA and A-PSCA groups ( $\mathrm{p}<0.001)$. In addition, we found that the most commonly selected recipient vessels in this study were the temporal PSCAs - with the majority of the blood supplied from the MCA or PCA (Table 1).

\section{Postoperative CHP and Hemodynamic Evaluation}

The representative image of the qualitative analysis of hemodynamic changes at the anastomosis site (Fig. 4) showed a high incidence of postoperative focal CHP (32/75, 42.7\%), including 14 patients with symptomatic CHP $(14 / 75,18.7 \%)$. The post- and preoperative rCBF values in the M-PSCA group were significantly higher than those in the non-M-PSCA group ( $<<0.001)$. The results of the multivariate analysis revealed that the hemodynamic source of the PSCAs from the MCA was significantly associated with the development of focal $(\mathrm{p}=$ $0.003)$ and symptomatic $(\mathrm{p}=0.021)$ CHP. Moreover, other factors such as age, sex, surgical side, onset, and bypass site were not associated with postoperative CHP (Table 2 ). The clinical symptoms of the patients with symptomatic CHP included small cerebral hematoma in 2 patients, moderate cerebral hematoma in 1 patient, intraoperative hemorrhage in 1 patient, motor weakness in 3 patients, aphasia in 7 patients, and seizure in 5 patients. The onset time varied from 0 to 15 days after surgery. Most of the patients completely recovered, except that 1 patient developed permanent neurological deficits and 1 patient died. Three of the 4 patients with perioperative hemorrhage recovered well with no residual neurological deficits, and 1 patient became disabled due to the secondary large infarction. One patient experienced motor aphasia and weakness of the right upper limb from day 1 to day 10, following an intractable seizure and secondary large infarction in both the frontal and parietal lobes despite strict blood pressure control, and that patient died on day 15 after surgery. Remarkably, $85.7 \%(12 / 14)$ of the patients with symptomatic CHP and all 4 (100\%) patients with postoperative hemorrhage were from the M-PSCA group.

\section{Discussion}

The PSCAs, which are commonly selected as the re-

TABLE 1. Basic characteristic analysis between the groups according to the hemodynamic sources of the recipient arteries

\begin{tabular}{|c|c|c|c|c|c|c|}
\hline \multirow[b]{3}{*}{ Characteristic } & \multicolumn{5}{|c|}{ Classification of Recipient Arteries According to Their Hemodynamic Sources } & \multirow[b]{3}{*}{ p Value } \\
\hline & \multirow[b]{2}{*}{ M-PSCAs $(n=45)$} & \multicolumn{4}{|c|}{ Non-M-PSCAs $(n=30)$} & \\
\hline & & A-PSCAs $(n=9)$ & CA-PSCAs $(n=4)$ & P-PSCAs $(n=15)$ & E-PSCAs $(n=2)$ & \\
\hline Age, yrs & $45.98 \pm 11.48$ & $47.78 \pm 14.33$ & $46.25 \pm 1.89$ & $44.87 \pm 11.77$ & $46.50 \pm 0.71$ & 0.985 \\
\hline Male sex & $22(48.9 \%)$ & $7(77.8 \%)$ & $2(50 \%)$ & $12(80 \%)$ & 0 & 0.067 \\
\hline Hemorrhagic/ischemic onset & $23 / 22$ & $2 / 7$ & $0 / 4$ & $6 / 9$ & $0 / 2$ & 0.118 \\
\hline Surgical side (lt/rt) & $20 / 25$ & $3 / 6$ & $1 / 3$ & $7 / 8$ & $1 / 1$ & 0.905 \\
\hline Suzuki stage & $3.22 \pm 0.85$ & $3.00 \pm 0.00$ & $4.75 \pm 0.50$ & $4.73 \pm 0.46$ & $5.00 \pm 0.00$ & $<0.001$ \\
\hline Postop/preop rCBF & $1.459 \pm 0.334$ & \multicolumn{4}{|c|}{$1.205 \pm 0.187$} & $<0.001$ \\
\hline
\end{tabular}

M-PSCAs = recipient PSCAs with blood flow from the MCA; $A-P S C A s=$ from the ACA; $C A-P S C A s=$ from the contralateral ACA; P-PSCAs = from the PCA; E-PSCAs = from the ECA; non-M-PSCAs = all recipient PSCAs with blood not supplied by the MCA.

Numbers for male sex, hemorrhagic/ischemic onset, and surgical side reflect the hemisphere counts, not the number of patients. 
Zhang et al.

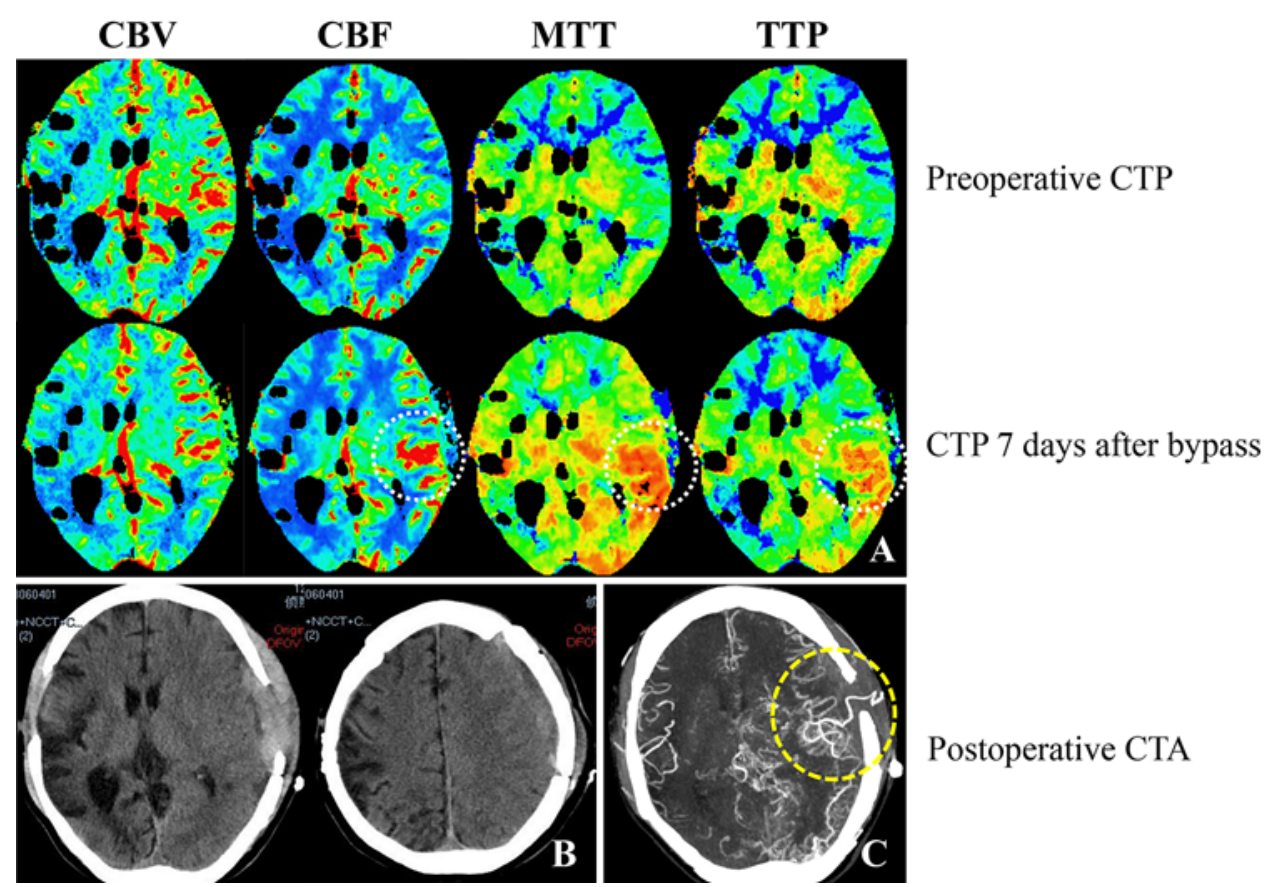

FIG. 4. Qualitative analysis of postoperative focal hyperperfusion. A: Qualitative evaluation of hemodynamic changes at the anastomosis site according to the CTP maps before and 7 days after bypass surgery. A focal increase in CBF was evident at the site of anastomosis from the postoperative CTP images (white circles). B: A regular postoperative CT scan demonstrated no hemorrhage or infarction in the hemisphere that underwent operation. C: Postoperative CTA showed a good patency of the anastomosis (yellow circle). $\mathrm{CBV}=$ cerebral blood volume; TTP = time to peak.

cipient vessels for direct anastomosis in patients with MMD, have blood supplies mainly from either the MCA or non-MCAs based on hemodynamic information from DSA. To the best of our knowledge, our study is the first in the literature to analyze the relationship between the hemodynamic sources of recipient PSCAs and postoperative CHP.

\section{Different Hemodynamic Sources of the PSCAs in Patients With MMD}

Our DSA data demonstrated that the distal branches of numerous MCAs $(20 / 75,26.7 \%)$ clearly did not reach the cortical surface, and the blood supply to the PSCAs was completely derived from non-MCAs due to the typical pia collateral circulation of patients with MMD. In this study,
$40 \%$ of selected recipient PSCAs had their major hemodynamic sources from non-MCAs.

Importantly, the distributional analysis of the hemodynamic origins showed that most of the frontal and parietal PSCAs were M-PSCAs and A-PSCAs, whereas the majority of the temporal PSCAs were M-PSCAs and P-PSCAs (Fig. 3). This observation is in accordance with the normal anatomical features of intracranial arteries, where the prior collateral source that compensates for decreases in MCA blood flow is the ACA in the frontal and parietal lobes and the PCA in the temporal lobe. ${ }^{6,18}$

Although there were no significant differences in age, sex, onset, or surgical side among the M-PSCA, A-PSCA, CA-PSCA, P-PSCA, and E-PSCA groups, the scores of Suzuki staging in the CA-PSCA, P-PSCA, and E-PSCA

TABLE 2. Multivariate analysis of postoperative cerebral hyperperfusion in STA-MCA bypass for MMD

\begin{tabular}{lccccccc}
\hline & \multicolumn{2}{c}{ Focal CHP } & & \multicolumn{2}{c}{ Symptomatic CHP } & \\
& Factor & Yes $(\mathrm{n}=32)$ & No $(\mathrm{n}=43)$ & $\mathrm{p}$ Value & Yes $(\mathrm{n}=14)$ & No $(\mathrm{n}=61)$ & $\mathrm{p}$ Value \\
\hline Age, yrs & $47.84 \pm 12.13$ & $44.63 \pm 10.57$ & 0.226 & $42.57 \pm 13.07$ & $46.79 \pm 10.82$ & 0.186 \\
\hline Male sex & $18(56.3 \%)$ & $25(58.1 \%)$ & 0.752 & $8(57.1 \%)$ & $35(57.4 \%)$ & 0.713 \\
\hline Hemorrhagic onset & $17(53.1 \%)$ & $14(32.6 \%)$ & 0.186 & $9(64.3 \%)$ & $22(36.1 \%)$ & 0.123 \\
\hline Surgical side (It) & $11(34.4 \%)$ & $21(48.8 \%)$ & 0.179 & $8(57.1 \%)$ & $24(39.3 \%)$ & 0.223 \\
\hline Bypass site (frontal/temporal/parietal lobe) & $8 / 21 / 3$ & $10 / 27 / 6$ & 0.955 & $2 / 10 / 2$ & $16 / 38 / 7$ & 0.242 \\
\hline M-PSCAs/non-M-PSCAs & $26 / 6$ & $19 / 24$ & 0.003 & $12 / 2$ & $33 / 28$ & 0.021 \\
\hline
\end{tabular}

Numbers for all factors except age reflect the hemisphere counts, not the number of patients. 
groups were substantially higher than those in the M-PSCA and A-PSCA groups ( $p<0.001$, Table 1 ). It is conceivable that patients with advanced MMD are prone to having a compromised ICA (MCA and ACA) with relatively strong non-ICA collaterals (contralateral ACA, PCA, and ECA) (Fig. 1). ${ }^{4,26}$

\section{Relationship Between the Hemodynamic Sources of Recipient PSCAs and Postoperative CHP}

Postoperative CHP after surgical revascularization commonly occurs in MMD. ${ }^{27}$ Its close relationship with postoperative complications, such as TNDs and intracerebral hemorrhage, has been studied in recent years. . $^{2,314,16 \text {, }}$ ${ }^{27,28}$ In adult patients with MMD with increases in cerebral blood volume preoperatively, a left-side operation and/or hemorrhagic onset were demonstrated to be risk factors for symptomatic postsurgery $\mathrm{CHP}$, but these factors cannot be used as accurate predictions for the occurrence of $\mathrm{CHP}^{2,3,27}$

The high incidence of postoperative focal CHP in this study $(42.7 \%)$ was in accordance with the results of previous studies. . $3,15,27$ Intriguingly, the increase in $\mathrm{rCBF}$ in the M-PSCA group was significantly higher than that in the non-M-PSCA group ( $\mathrm{p}<0.001$ ); moreover, the hemodynamic source of PSCAs from the MCA was significantly associated with the development of focal CHP $(p=0.003)$ (Table 2). The blood supply of M-PSCAs normally came from a severe stenotic or moyamoya-like MCA, whereas most of the non-M-PSCAs had normal hemodynamic origins. This discrepancy would generally result in a low blood flow velocity and pressure in M-PSCAs, which is in contrast to those in non-M-PSCAs. It is conceivable that anastomosis of M-PSCAs can cause greater postoperative hemodynamic changes than that of non-M-PSCAs. Furthermore, the network formation of M-PSCAs may be unstable, and the cerebral cortex perfused by each artery may be more compartmentalized in M-PSCAs than in non-M-PSCAs. These phenomena may cause the postoperative hemodynamic distribution to be restricted to a relatively small area immediately after the bypass. ${ }^{2}$ Thus, anastomosis of M-PSCAs is more likely to be related to focal CHP. However, the condition of the donor artery, such as the STA, should also be considered. Ishii et al. reported a more than 1.5-fold dilatation of the STA in the acute postoperative stage, and this dilatation was significantly correlated with TNDs. ${ }^{11}$ In addition, another study demonstrated that the caliber of the STA largely determined blood flow immediately after STA-MCA anastomosis. ${ }^{27}$ Together, these results suggest that patients who did not have focal CHP in the M-PSCA group might have a relatively small hemodynamic discrepancy between the STA and M-PSCAs. Future studies are needed to determine the significance of this discrepancy for predicting focal CHP.

The analysis of focal CHP is divided into symptomatic CHP and silent CHP in most reported studies. ${ }^{2,3,13,15,27}$ In adult patients with MMD the incidence of postoperative symptomatic CHP varies considerably, from $16.7 \%$ to $38.2 \%, 1,3,13$ because of the lack of proper quantitative evaluations of postoperative $\mathrm{CBF}$ and different study populations. The incidence of symptomatic CHP (18.7\%) based on the qualitative CTP analysis in our study was similar to previously reported results..$^{1,3,13}$ Although the etiology of symptomatic CHP is still unknown, the quality of the recipient artery and its deep vascular structure may play a critical role in the occurrence of symptomatic CHP. In occlusive cerebrovascular diseases, especially in MMD, cerebrovascular resistance decreases, and distal vascular diameter maximally dilates. ${ }^{21,22}$ These changes can cause the arterial wall to bear strong stress and become thin and fragile.

According to one of our previous studies, high expression of hypoxia-inducible COX-2 and mPGES-1 in the cortical arterial wall may increase vascular permeability, decrease vascular tone, and inhibit the rapid repair of vascular wall injury. ${ }^{29}$ In the present study, multivariate analysis revealed that the hemodynamic source of PSCAs from the MCA was significantly associated with the development of symptomatic CHP ( $p=0.021)$ (Table 2). Moreover, our data remarkably showed that $12(85.7 \%)$ of the 14 patients with symptomatic CHP and all 4 (100\%) patients with postoperative hemorrhage were from the M-PSCA group. Together, these findings suggest that the recipient artery under long-term ischemic and hypoxic conditions, such as PSCAs with hemodynamic sources from the MCA, is likely to have a weak vascular quality and to be vulnerable to sudden increases in focal blood flow after bypass surgery.

\section{Limitations of the Study}

Our preliminary study is limited by its retrospective nature, and it contains a small number of patients with a rare disease. In addition, it is a single-center study, and the selection bias of the sample could be an issue. For evaluating the hemodynamic changes between the pre- and postoperative hemispheres or the diagnosis of CHP, the CTP used in this study was not better than the use of SPECT.

\section{Conclusions}

Our study demonstrates that direct anastomoses of PSCAs with anterograde hemodynamic sources from the MCA have a much higher risk of postoperative $\mathrm{CHP}$ than those from non-MCAs in adult patients with MMD. Identifying the hemodynamic sources of PSCAs from the MCA or non-MCAs may help to predict the occurrence of postoperative CHP.

\section{Acknowledgments}

This study was partly supported by the project of the National Natural Science Foundation of China (81671157).

\section{References}

1. Fujimura M, Kaneta T, Mugikura S, Shimizu H, Tominaga $\mathrm{T}$ : Temporary neurologic deterioration due to cerebral hyperperfusion after superficial temporal artery-middle cerebral artery anastomosis in patients with adult-onset moyamoya disease. Surg Neurol 67:273-282, 2007

2. Fujimura M, Niizuma K, Endo H, Sato K, Inoue T, Shimizu $\mathrm{H}$, et al: Quantitative analysis of early postoperative cerebral blood flow contributes to the prediction and diagnosis of cerebral hyperperfusion syndrome after revascularization surgery for moyamoya disease. Neurol Res 37:131-138, 2015

3. Fujimura M, Shimizu H, Inoue T, Mugikura S, Saito A, Tom- 
inaga T: Significance of focal cerebral hyperperfusion as a cause of transient neurologic deterioration after extracranialintracranial bypass for moyamoya disease: comparative study with non-moyamoya patients using $\mathrm{N}$-isopropyl-p-[ $\left.{ }^{123} \mathrm{I}\right]$ iodoamphetamine single-photon emission computed tomography. Neurosurgery 68:957-965, 2011

4. Fujimura M, Tominaga T: Current status of revascularization surgery for Moyamoya disease: special consideration for its 'internal carotid-external carotid (IC-EC) conversion' as the physiological reorganization system. Tohoku J Exp Med 236:45-53, 2015

5. Fukui M: Guidelines for the diagnosis and treatment of spontaneous occlusion of the circle of Willis ('moyamoya' disease). Research Committee on Spontaneous Occlusion of the Circle of Willis (Moyamoya Disease) of the Ministry of Health and Welfare, Japan. Clin Neurol Neurosurg 99 (Suppl 2):S238-S240, 1997

6. Geri O, Shiran SI, Roth J, Artzi M, Ben-Sira L, Ben Bashat $\mathrm{D}$ : Vascular territorial segmentation and volumetric blood flow measurement using dynamic contrast enhanced magnetic resonance angiography of the brain. J Cereb Blood Flow Metab 37:3446-3456, 2017

7. Guzman R, Lee M, Achrol A, Bell-Stephens T, Kelly M, Do $\mathrm{HM}$, et al: Clinical outcome after 450 revascularization procedures for moyamoya disease. Clinical article. J Neurosurg 111:927-935, 2009

8. Haubrich C, Kruska W, Diehl RR, Möller-Hartmann W, Klötzsch C: Dynamic autoregulation testing in patients with middle cerebral artery stenosis. Stroke 34:1881-1885, 2003

9. Hayashi T, Shirane R, Fujimura M, Tominaga T: Postoperative neurological deterioration in pediatric moyamoya disease: watershed shift and hyperperfusion. J Neurosurg Pediatr 6:73-81, 2010

10. Houkin K, Ishikawa T, Yoshimoto T, Abe H: Direct and indirect revascularization for moyamoya disease surgical techniques and peri-operative complications. Clin Neurol Neurosurg 99 (Suppl 2):S142-S145, 1997

11. Ishii D, Okazaki T, Matsushige T, Shinagawa K, Ichinose N, Sakamoto S, et al: Postoperative dilatation of superficial temporal artery associated with transient neurologic symptoms after direct bypass surgery for moyamoya angiopathy. World Neurosurg 106:435-441, 2017

12. Januszewski J, Beecher JS, Chalif DJ, Dehdashti AR: Flowbased evaluation of cerebral revascularization using nearinfrared indocyanine green videoangiography. Neurosurg Focus 36(2):E14, 2014

13. Kaku Y, Iihara K, Nakajima N, Kataoka H, Fukuda K, Masuoka J, et al: Cerebral blood flow and metabolism of hyperperfusion after cerebral revascularization in patients with moyamoya disease. J Cereb Blood Flow Metab 32:20662075,2012

14. Kashiwazaki D, Akioka N, Kuwayama N, Houkin K, Czabanka M, Vajkoczy P, et al: Berlin grading system can stratify the onset and predict perioperative complications in adult moyamoya disease. Neurosurgery 81:986-991, 2017

15. Kazumata K, Uchino H, Tokairin K, Ito M, Shiga T, Osanai $\mathrm{T}$, et al: Cerebral hyperperfusion syndrome after revascularization surgery in moyamoya disease: region-symptom mapping and estimating a critical threshold. World Neurosurg 114:e388-e395, 2018

16. Kim JE, Oh CW, Kwon OK, Park SQ, Kim SE, Kim YK: Transient hyperperfusion after superficial temporal artery/ middle cerebral artery bypass surgery as a possible cause of postoperative transient neurological deterioration. Cerebrovasc Dis 25:580-586, 2008

17. Ley-Pozo J, Willmes K, Ringelstein EB: Relationship between pulsatility indices of Doppler flow signals and $\mathrm{CO}_{2}-$ reactivity within the middle cerebral artery in extracranial occlusive disease. Ultrasound Med Biol 16:763-772, 1990
18. Liebeskind DS: Collateral circulation. Stroke 34:2279-2284, 2003

19. Meyer IA, Cereda CW, Correia PN, Zerlauth JB, Puccinelli F, Rotzinger DC, et al: Factors associated with focal computed tomographic perfusion abnormalities in supratentorial transient ischemic attacks. Stroke 49:68-75, 2018

20. Miyamoto S, Yoshimoto T, Hashimoto N, Okada Y, Tsuji I, Tominaga $\mathrm{T}$, et al: Effects of extracranial-intracranial bypass for patients with hemorrhagic moyamoya disease: results of the Japan Adult Moyamoya Trial. Stroke 45:1415-1421, 2014

21. Morisawa H, Kawamata T, Kawashima A, Hayashi M, Yamaguchi K, Yoneyama T, et al: Hemodynamics and changes after STA-MCA anastomosis in moyamoya disease and atherosclerotic cerebrovascular disease measured by microDoppler ultrasonography. Neurosurg Rev 36:411-419, 2013

22. Nair AK, Drazin D, Yamamoto J, Boulos AS: Computed tomographic perfusion in assessing postoperative revascularization in moyamoya disease. World Neurosurg 73:93-99, e13, 2010

23. Okada Y, Shima T, Nishida M, Yamane K, Yamada T, Yamanaka C: Effectiveness of superficial temporal artery-middle cerebral artery anastomosis in adult moyamoya disease: cerebral hemodynamics and clinical course in ischemic and hemorrhagic varieties. Stroke 29:625-630, 1998

24. Research Committee on the Pathology and Treatment of Spontaneous Occlusion of the Circle of Willis: Guidelines for diagnosis and treatment of moyamoya disease (spontaneous occlusion of the circle of Willis). Neurol Med Chir (Tokyo) 52:245-266, 2012

25. Scott RM, Smith ER: Moyamoya disease and moyamoya syndrome. N Engl J Med 360:1226-1237, 2009

26. Suzuki J, Takaku A: Cerebrovascular "moyamoya" disease. Disease showing abnormal net-like vessels in base of brain. Arch Neurol 20:288-299, 1969

27. Uchino H, Kuroda S, Hirata K, Shiga T, Houkin K, Tamaki $\mathrm{N}$ : Predictors and clinical features of postoperative hyperperfusion after surgical revascularization for moyamoya disease: a serial single photon emission CT/positron emission tomography study. Stroke 43:2610-2616, 2012

28. Uchino H, Nakayama N, Kazumata K, Kuroda S, Houkin $\mathrm{K}$ : Edaravone reduces hyperperfusion-related neurological deficits in adult moyamoya disease: historical control study. Stroke 47:1930-1932, 2016

29. Zhang J, Xiong Z, Wang S, He Y, Sun S, Wu X, et al: Cyclooxygenase- 2 and prostaglandin $\mathrm{E} 2$ are associated with middle cerebral artery occlusion and hemorrhage in patients with moyamoya disease. Curr Neurovasc Res 13:68-74, 2016

\section{Disclosures}

The authors report no conflict of interest concerning the materials or methods used in this study or the findings specified in this paper.

\section{Author Contributions}

Conception and design: Chen, Zhang, Fujimura, Lau. Acquisition of data: $\mathrm{S} \mathrm{Li}, \mathrm{Wu}, \mathrm{Hu}$, Zheng. Analysis and interpretation of data: Chen, Zhang, Xu, Zhao. Drafting the article: Zhang. Critically revising the article: Chen, Zhang, Zhao, X Li. Reviewed submitted version of manuscript: Chen, Zhang, Fujimura, Lau, $\mathrm{X} \mathrm{Li}$. Approved the final version of the manuscript on behalf of all authors: Chen. Statistical analysis: Zhang, Hu. Administrative/technical/material support: Chen. Study supervision: Chen, Zhang.

\section{Correspondence}

Jincao Chen: Zhongnan Hospital of Wuhan University, Wuhan, China.chenjincao2012@hotmail.com. 